Research Article

\title{
A Diophantine Problem with Unlike Powers of Primes
}

\author{
Quanwu Mu (D) and Liyan Xi \\ School of Science, Xi'an Polytechnic University, Xi'an, 710048, Shaanxi, China \\ Correspondence should be addressed to Quanwu Mu; muquanwu@163.com
}

Received 10 January 2021; Revised 10 February 2021; Accepted 22 February 2021; Published 8 March 2021

Academic Editor: Tingting Wang

Copyright (c) 2021 Quanwu Mu and Liyan Xi. This is an open access article distributed under the Creative Commons Attribution License, which permits unrestricted use, distribution, and reproduction in any medium, provided the original work is properly cited.

Let $k$ be an integer with $4 \leq k \leq 6$ and $\eta$ be any real number. Suppose that $\lambda_{1}, \lambda_{2}, \ldots, \lambda_{5}$ are nonzero real numbers, not all of them have the same sign, and $\lambda_{1} / \lambda_{2}$ is irrational. It is proved that the inequality $\left|\lambda_{1} p_{1}+\lambda_{2} p_{2}^{2}+\lambda_{3} p_{3}^{3}+\lambda_{4} p_{4}^{4}+\lambda_{5} p_{5}^{k}+\eta\right|<\left(\max _{1 \leq j \leq 5} p_{j}\right)^{-\sigma(k)}$ has infinitely many solutions in prime variables $p_{1}, p_{2}, p_{3}, p_{4}$, and $p_{5}$, where $0<\sigma(4)<1 / 36,0<\sigma(5)<4 / 189$, and $0<\sigma(6)<1 / 54$. This gives an improvement of the recent results.

\section{Introduction}

The determination of the minimal $s$ such that the Diophantine equation

$$
N=\sum_{i=1}^{s} x_{i}^{i+1}
$$

is solvable in positive integers $x_{1}, \ldots, x_{s}$, for all sufficiently large integers $N$ is an interesting problem in additive number theory. In 1951, Roth [1] proved that $s=50$ is acceptable. This result was subsequently improved by Thanigasalam et al. [2-4], Vaughan and Vaughan $[5,6]$, Brüdern and Brüdern $[7,8]$ and Ford and Ford $[9,10]$. The best currently known result is due to Ford [10], with $s=14$. Schwarz [11] suggested to analyze the related Diophantine inequality. The first result was obtained by Brüdern [12], who showed that the values of

$$
\sum_{i=1}^{22} \lambda_{i+1} x_{i}^{i+1},
$$

at integer points $\left(x_{1}, \ldots, x_{22}\right)$ are dense on the real line provided that $\lambda_{2}, \ldots, \lambda_{23}$ are nonzero real numbers and $\lambda_{2} / \lambda_{3}$ is irrational. Thanks to a pruning technique, Brüdern [13] proved that the values taken by

$$
\sum_{i=1}^{16} \lambda_{i+1} x_{i}^{i+1}
$$

at integer points $\left(x_{1}, \ldots, x_{16}\right)$ are dense on the real line if $\lambda_{2}, \ldots, \lambda_{17}$ are nonzero real numbers and at least one of the ratios $\lambda_{i} / \lambda_{j}$ is irrational.

Suppose that $x_{1}, \ldots, x_{s}$ are prime variables, $N$ is a sufficiently large integer, and $N+s$ is even. In 1969, Vaughan proved in his doctoral thesis that (1) is solvable if $s=31$. Later, Vaughan [5] improved upon his own result by taking $s=30$ in place of $s=31$. By calculating, the exponential density more accurately, Shan [14] showed that $s=$ 23 is acceptable. In addition, Prachar [15] established that each sufficiently large odd integer $N$ can be represented as

$$
N=p_{1}+p_{2}^{2}+p_{3}^{3}+p_{4}^{4}+p_{5}^{5}
$$

where $p_{1}, p_{2}, \ldots, p_{5}$ are prime numbers. As a corollary of [16] in Theorem 1, Ren and Tsang obtained the same result as Prachar. It is of some interest to consider the analogous form for Diophantine inequalities. Let $\lambda_{1}, \lambda_{2}, \ldots, \lambda_{5}$ be nonzero real numbers, not all of them have the same sign and $\lambda_{1} / \lambda_{2}$ as irrational. In 2016, Ge and $\mathrm{Li}$ [17] proved that, for any given real numbers $\eta$ and $\sigma, 0<\sigma<1 / 720$, there exist infinitely many solutions in prime numbers $p_{j}$ to the inequality 


$$
\left|\lambda_{1} p_{1}+\lambda_{2} p_{2}^{2}+\lambda_{3} p_{3}^{3}+\lambda_{4} p_{4}^{4}+\lambda_{5} p_{5}^{5}+\eta\right|<\left(\max _{1 \leq j \leq 5} p_{j}^{j}\right)^{-\sigma} .
$$

Let $k \geq 4$ be an integer. The first author [18] investigated the solvability of more general Diophantine inequality

$$
\left|\lambda_{1} p_{1}+\lambda_{2} p_{2}^{2}+\lambda_{3} p_{3}^{3}+\lambda_{4} p_{4}^{4}+\lambda_{5} p_{5}^{k}+\eta\right|<\left(\max _{1 \leq j \leq 5} p_{j}\right)^{-\sigma(k)}
$$

and proved that (6) has infinitely many solutions in prime variables $p_{j}$ for $0<\sigma(4)<5 / 288$ and $0<\sigma(k)<5 /\left(6 k^{2}(k+\right.$ 1)) with $k \geq 5$. Subsequently, Liu [19] obtained $0<\sigma(5)<5 / 288$. In [20], the first author and Qu showed that $0<\sigma(5)<5 / 252$ is acceptable. Very recently, this result was improved by Zhu [21], who obtained $0<\sigma(5)<1 / 48$. In [22], Gao and Liu gave an improvement ([18] in Theorem 1.2) in case $k \geq 6$, and they proved $0<\sigma(6)<1 / 56$ particularly.

The main purpose of this paper is to sharpen the above results in case $4 \leq k \leq 6$. We obtain the following theorem.

Theorem 1. Let $k$ be an integer with $4 \leq k \leq 6$ and $\eta$ be any given real number. Suppose that $\lambda_{1}, \lambda_{2}, \ldots, \lambda_{5}$ are nonzero real numbers, not all of them are same sign, and $\lambda_{1} / \lambda_{2}$ is irrational. Then, inequality (6) has infinitely many solutions in prime variables $p_{1}, p_{2}, \ldots, p_{5}$, where $0<\sigma(4)<1 / 36,0<\sigma(5)<4 / 189$, and $0<\sigma(6)<1 / 54$.

The improvement derives not only from the use of the function $\rho(m)$ constructed by Harman and Kumchev (see Section 8 in [23] and Section 5 in [24], for details) but also from some ingredients in [21]. It is worth remarking that $\mathrm{Ge}$ et al. [25] obtained $0<\sigma(5)<1 / 32$, if the condition " $\lambda_{1} / \lambda_{2}$ is irrational" in Theorem 1 is replaced by " $\lambda_{1} / \lambda_{2}$ is irrational and $\lambda_{2} / \lambda_{4}$ and $\lambda_{3} / \lambda_{5}$ are rational."

Notation. Throughout the paper, $\varepsilon$ and $\delta$ are arbitrarily small, fixed positive real numbers. Any statement in which $\varepsilon$ occurs holds for each positive $\varepsilon$. The implicit constants in O-term, «- and 》-symbols depend at most on $\lambda_{1}, \lambda_{2}, \ldots, \lambda_{5}$ and $\varepsilon$. The letter $p$, with or without subscript, is reserved for a prime number. By $A \asymp B$, we mean that $A \ll B$ and $A \gg B$. For simplicity, we write $\mathscr{L}=\log X$ and $e(\alpha)=\exp (2 \pi i \alpha)$.

\section{Preliminaries}

We apply the Davenport-Heilbronn circle method (see [26] and Chapter 11 in [27]) to prove Theorem 1 . Since $\lambda_{1} / \lambda_{2}$ is irrational, there are infinitely many convergents to its continued fraction. Let $q$ be any denominator of a convergent to $\lambda_{1} / \lambda_{2}$. As in [20], let $X$ run through the sequences:

$$
X=q^{(7 / 3)} .
$$

We set

$$
\begin{aligned}
\mathscr{I} & =\left[\frac{1}{3} X^{(1 / 2)}, \frac{2}{3} X^{(1 / 2)}\right), \\
S_{2}^{*}(\alpha) & =\sum_{m \in \mathscr{I}} \rho(m) e\left(\alpha m^{2}\right),
\end{aligned}
$$

where the function $\rho(m)$ is defined by 5.2 in [24]. According to [24], $\rho(m)$ is a nontrivial lower bound for the characteristic function of the set of primes in $\mathscr{I}$, and it satisfies

$$
\rho(m) \leq \begin{cases}1, & \text { if } m \text { is a prime, } \\ 0, & \text { otherwise. }\end{cases}
$$

For further properties of $\rho(m)$, see Lemma 1 and (4.2)-(4.4) in [24]. Let

$$
\begin{aligned}
I_{j} & =\left[(\delta X)^{(1 / j)}, X^{(1 / j)}\right], \\
S_{j}(\alpha) & =\sum_{p \in I_{j}}(\log p) e\left(\alpha p^{j}\right) .
\end{aligned}
$$

By the prime number theorem, it is easy to show that $S_{j}(\alpha)$ $\ll X^{(1 / j)}$. For any fixed $\tau>0$, set $K_{\tau}(\alpha)=(\pi \alpha)^{-2} \sin ^{2}(\pi \tau \alpha)$ for $\alpha \neq 0$ and $K_{\tau}(0)=\tau^{2}$. Clearly, we have

$$
K_{\tau}(\alpha) \ll \min \left(\left(\tau^{2},|\alpha|^{-2}\right) .\right.
$$

A straightforward application of the Cauchy integral formula gives

$$
\int_{-\infty}^{+\infty} e(x \alpha) K_{\tau}(\alpha) \mathrm{d} \alpha=\max (0, \tau-|x|)
$$

Identity (12) is also a corollary of Lemma 4 in [26]. For $4 \leq k \leq 6$, put

$$
G(\alpha)=S_{1}\left(\lambda_{1} \alpha\right) S_{2}^{*}\left(\lambda_{2} \alpha\right)\left(\prod_{j=3}^{4} S_{j}\left(\lambda_{j} \alpha\right)\right) S_{k}\left(\lambda_{5} \alpha\right) e(\alpha \eta) K_{\tau}(\alpha) .
$$

We write

$$
I(\tau, \eta, \mathfrak{X})=\int_{\mathfrak{X}} G(\alpha) \mathrm{d} \alpha,
$$

for any measurable subset $\mathfrak{X}$ of $\mathbb{R}$. It follows from (9) and (12) that

$$
\begin{aligned}
I(\tau, \eta, \mathbb{R})= & \sum_{\substack{p_{j} \in I_{j}, j=1,3,4, p_{5} \in I_{k}, m_{2} \in \mathcal{F}}} \rho\left(m_{2}\right) \prod_{1 \leq j \leq 5} \log p_{j} \\
& \times \int_{-\infty}^{+\infty} e\left(\left(\lambda_{1} p_{1}+\lambda_{2} m_{2}^{2}+\sum_{j=3}^{4} \lambda_{j} p_{j}^{j}+\lambda_{5} p_{5}^{k}+\eta\right) \alpha\right) \\
K_{\tau}(\alpha) \mathrm{d} \alpha \quad & \sum_{p_{j} \in I_{j}, j=1,3,4} \rho\left(m_{2}\right) \prod_{1 \leq j \leq 5} \log p_{j} \\
& p_{5} \in I_{k}, m_{2} \in \mathcal{F} \\
& \times \max \left(0, \tau-\left|\lambda_{1} p_{1}+\lambda_{2} m_{2}^{2}+\sum_{j=3}^{4} \lambda_{j} p_{j}^{j}+\lambda_{5} p_{5}^{k}+\eta\right|\right) \\
\leq & \tau \mathscr{L}^{4} \mathcal{N}(X),
\end{aligned}
$$


where $\mathcal{N}(X)$ denotes the number of solutions of the inequality

$$
\left|\lambda_{1} p_{1}+\lambda_{2} p_{2}^{2}+\lambda_{3} p_{3}^{3}+\lambda_{4} p_{4}^{4}+\lambda_{5} p_{5}^{k}+\eta\right|<\tau,
$$

with $p_{2} \in \mathscr{I}, p_{5} \in I_{k}$, and $p_{j} \in I_{j}$ for $j \in\{1,3,4\}$. In what follows, we take

$$
\tau= \begin{cases}X^{-(1 / 36)+20 \varepsilon} & \text { if } k=4, \\ X^{-(4 / 189)+20 \varepsilon} & \text { if } k=5, \\ X^{-(1 / 54)+20 \varepsilon} & \text { if } k=6,\end{cases}
$$

actually. We now divide the real line into three disjoint parts:

$$
\begin{aligned}
\mathfrak{M} & =\left\{\alpha:|\alpha| \leq X^{-(1 / 8)}\right\}, \\
\mathfrak{m} & =\left\{\alpha: X^{-(1 / 8)}<|\alpha| \leq \xi\right\}, \\
\mathfrak{t} & =\{\alpha:|\alpha|>\xi\},
\end{aligned}
$$

where $\xi=\tau^{-2} X^{(1 / 16)-(1 / 4 k)+10 \varepsilon}$. These sets are called the major arc, the minor arcs, and the trivial regions, respectively.

In the following sections, we shall prove that the dominant contribution to $I(\tau, \eta, \mathbb{R})$ is from the major arc, and the contribution from the minor arcs and the trivial region can be neglected.

\section{The Major Arc}

Our first goal is to show that

$$
|I(\tau, \eta, \mathfrak{M})| \gg \tau^{2} X^{(13 / 12)+(1 / k)} \mathscr{L}^{-1} .
$$

The proof of (19) is quite similar to that given in Section 3 in [20]. For completeness of exposition, we briefly present the proof procedure below.

Let

$$
\begin{aligned}
& \mathfrak{M}_{1}=\left\{\alpha:|\alpha| \leq X^{-1+(5 / 12 k)-\varepsilon}\right\}, \\
& \mathfrak{M}_{2}=\left\{\alpha: X^{-1+(5 / 12 k)-\varepsilon}<|\alpha| \leq X^{-(7 / 8)}\right\}, \\
& \mathfrak{M}_{3}=\left\{\alpha: X^{-(7 / 8)}<|\alpha| \leq X^{-(1 / 8)}\right\} .
\end{aligned}
$$

Then, we have $\mathfrak{M}=\mathfrak{M}_{1} \cup \mathfrak{M}_{2} \cup \mathfrak{M}_{3}$ and

$I(\tau, \eta, \mathfrak{M})=I\left(\tau, \eta, \mathfrak{M}_{1}\right)+I\left(\tau, \eta, \mathfrak{M}_{2}\right)+I\left(\tau, \eta, \mathfrak{M}_{3}\right)$.
By a similar argument as that in pp. 1656-1657 in [20], we can obtain

$$
\left|I\left(\tau, \eta, \mathfrak{M}_{1}\right)\right| \gg \tau^{2} X^{(13 / 12)+(1 / k)} \mathscr{L}^{-1} .
$$

To estimate the integrals $I\left(\tau, \eta, \mathfrak{M}_{2}\right)$ and $I\left(\tau, \eta, \mathfrak{M}_{3}\right)$, we need the following two lemmas.

Lemma 1. Let $j \geq 2$ be an integer. Then, for nonzero real number $\lambda$ and any $\varepsilon>0$, we have

$$
S_{j}(\lambda \alpha) \ll\left\{\begin{array}{l}
X^{(1 / j)\left(1-j \cdot 4^{1-j}\right)+\varepsilon}|\alpha|^{-4^{1-j}} \text { for } X^{-1} \leq|\alpha| \leq X^{-1+(1 / 2 j)}, \\
X^{\frac{1}{j}\left(1-(1 / 2) \cdot 4^{1-j}\right)+\varepsilon} \text { for } X^{-1+(1 / 2 j)}<|\alpha| \leq X^{-(1 / 2 j) .}
\end{array}\right.
$$

Proof. It follows from Theorem 1 in [28].

Lemma 2. For $4 \leq k \leq 6$, suppose that

$$
\begin{aligned}
& F(\alpha) \in\left\{S_{1}^{2}\left(\lambda_{1} \alpha\right), S_{3}^{8}\left(\lambda_{3} \alpha\right),\left(S_{2}^{*}\left(\lambda_{2} \alpha\right)\right)^{2} S_{3}^{4}\left(\lambda_{3} \alpha\right),\right. \\
&\left(S_{2}^{*}\left(\lambda_{2} \alpha\right)\right)^{2} S_{4}^{4}\left(\lambda_{4} \alpha\right), \\
&\left(S_{2}^{*}\left(\lambda_{2} \alpha\right)\right)^{2} S_{5}^{6}\left(\lambda_{5} \alpha\right),\left(S_{2}^{*}\left(\lambda_{2} \alpha\right)\right)^{2} S_{6}^{8}\left(\lambda_{5} \alpha\right), \\
&\left.\left(S_{2}^{*}\left(\lambda_{2} \alpha\right) S_{3}\left(\lambda_{3} \alpha\right) S_{k}\left(\lambda_{5} \alpha\right)\right)^{2},\left(S_{2}^{*}\left(\lambda_{2} \alpha\right)\right)^{2} S_{4}^{2}\left(\lambda_{4} \alpha\right) S_{5}^{4}\left(\lambda_{5} \alpha\right)\right\} .
\end{aligned}
$$

Then, we have

$$
\begin{gathered}
\int_{-1}^{1}|F(\alpha)| \mathrm{d} \alpha \ll X^{-1} F(0)^{1+\varepsilon}, \\
\int_{\mathbb{R}}|F(\alpha)| K_{\tau}(\alpha) \mathrm{d} \alpha \ll \tau X^{-1} F(0)^{1+\varepsilon} .
\end{gathered}
$$

Proof. See Lemma 3.7 in [20].

When $\alpha \in \mathfrak{M}_{2}$, it follows from (23) that

$$
S_{4}\left(\lambda_{4} \alpha\right) \ll X^{(15 / 64)+\varepsilon}|\alpha|^{-(1 / 64)} \ll X^{(1 / 4)-(5 / 768 k)+2 \varepsilon} \text {. }
$$

Combining this with the Cauchy-Schwarz inequality and Lemma 2 gives 


$$
\begin{aligned}
\left|I\left(\tau, \eta, \mathfrak{M}_{2}\right)\right| \ll & \tau^{2} \sup _{\alpha \in \mathfrak{M}_{2}}\left|S_{4}\left(\lambda_{4} \alpha\right)\right| \int_{\mathfrak{M}_{2}}\left|S_{1}\left(\lambda_{1} \alpha\right) S_{2}^{*}\left(\lambda_{2} \alpha\right) S_{3}\left(\lambda_{3} \alpha\right) S_{k}\left(\lambda_{5} \alpha\right)\right| \mathrm{d} \alpha \\
& \ll \tau^{2} \sup _{\alpha \in \mathfrak{M}_{2}}\left|S_{4}\left(\lambda_{4} \alpha\right)\right|\left(\int_{-1}^{1}\left|S_{1}\left(\lambda_{1} \alpha\right)\right|^{2} \mathrm{~d} \alpha\right)^{(1 / 2)} \\
& \times\left(\int_{-1}^{1}\left|S_{2}^{*}\left(\lambda_{2} \alpha\right) S_{3}\left(\lambda_{3} \alpha\right) S_{k}\left(\lambda_{5} \alpha\right)\right|^{2} d \alpha\right)^{(1 / 2)} \\
& \ll \tau^{2} X^{(1 / 4)-(5 / 768 k)+2 \varepsilon} \cdot\left(X^{1+2 \varepsilon}\right)^{(1 / 2)} \cdot\left(X^{(2 / 3)+(2 / k)+\varepsilon}\right)^{(1 / 2)} \\
& \ll \tau^{2} X^{(13 / 12)+(1 / k)-\varepsilon}
\end{aligned}
$$

where (11) is used.

When $\alpha \in \mathfrak{M}_{3}$, (23) implies

$$
S_{4}\left(\lambda_{4} \alpha\right) \ll X^{(1 / 4)-(1 / 512)+\varepsilon}
$$

Proceeding as in the proof of (27), we have

$$
\left|I\left(\tau, \eta, \mathfrak{M}_{3}\right)\right| \ll \tau^{2} X^{(13 / 12)+(1 / k)-\varepsilon} .
$$

This with (27), (22), and (21) yields (19).

\section{The Minor Arcs}

The next thing to do in the proof is to establish that

$$
|I(\tau, \eta, \mathfrak{m})| \ll \tau^{2} X^{(13 / 12)+(1 / k)} \mathscr{L}^{-2} .
$$

This work forms the bulk of the present paper. We subdivide $\mathfrak{m}$ into four disjoint parts: $\mathfrak{m}=\mathfrak{m}_{1} \cup \mathfrak{m}_{2} \cup \mathfrak{m}_{3} \cup \mathfrak{m}_{4}$, where

$$
\begin{aligned}
& \mathfrak{m}_{1}=\left\{\alpha \in \mathfrak{m}:\left|S_{1}\left(\lambda_{1} \alpha\right)\right| \leq X^{(6 / 7)+2 \varepsilon}\right\}, \\
& \mathfrak{m}_{2}=\left\{\alpha \in \mathfrak{m}:\left|S_{1}\left(\lambda_{1} \alpha\right)\right|>X^{(6 / 7)+2 \varepsilon},\left|S_{2}^{*}\left(\lambda_{2} \alpha\right)\right| \leq X^{(3 / 7)+2 \varepsilon},\left|S_{3}\left(\lambda_{3} \alpha\right)\right|>X^{(11 / 36)+2 \varepsilon}\right\}, \\
& \mathfrak{m}_{3}=\left\{\alpha \in \mathfrak{m}:\left|S_{1}\left(\lambda_{1} \alpha\right)\right|>X^{(6 / 7)+2 \varepsilon},\left|S_{2}^{*}\left(\lambda_{2} \alpha\right)\right| \leq X^{(3 / 7)+2 \varepsilon},\left|S_{3}\left(\lambda_{3} \alpha\right)\right| \leq X^{(11 / 36)+2 \varepsilon}\right\}, \\
& \mathfrak{m}_{4}=\left\{\alpha \in \mathfrak{m}:\left|S_{1}\left(\lambda_{1} \alpha\right)\right|>X^{(6 / 7)+2 \varepsilon},\left|S_{2}^{*}\left(\lambda_{2} \alpha\right)\right|>X^{(3 / 7)+2 \varepsilon}\right\} .
\end{aligned}
$$

Therefore,

$$
I(\tau, \eta, \mathfrak{m})=\sum_{j=1}^{4} I\left(\tau, \eta, \mathfrak{m}_{j}\right) .
$$

To prove (30), it suffices to show that $\left|I\left(\tau, \eta, \mathfrak{m}_{j}\right)\right| \ll \tau^{2} X^{(13 / 12)+(1 / k)} \mathscr{L}^{-2}$ holds for $1 \leq j \leq 4$.

We apply Hölder's inequality and Lemma 2 to estimate $\left|I\left(\tau, \eta, \mathfrak{m}_{1}\right)\right|$. When $k=4$, we have

$$
\begin{aligned}
& \left|I\left(\tau, \eta, \mathbf{m}_{1}\right)\right| \\
& \ll\left(\sup _{\alpha \in \mathfrak{m}_{1}}\left|S_{1}\left(\lambda_{1} \alpha\right)\right|\right)^{(1 / 4)}\left(\int_{\mathbb{R}}\left|S_{1}\left(\lambda_{1} \alpha\right)\right|^{2} K_{\tau}(\alpha) \mathrm{d} \alpha\right)^{(3 / 8)} \\
& \times\left(\int_{\mathbb{R}}\left|S_{3}\left(\lambda_{3} \alpha\right)\right|^{8} K_{\tau}(\alpha) \mathrm{d} \alpha\right)^{(1 / 8)}\left(\int_{\mathbb{R}}\left|S_{2}^{*}\left(\lambda_{2} \alpha\right)\right|^{2}\left|S_{4}\left(\lambda_{4} \alpha\right)\right|^{4} K_{\tau}(\alpha) \mathrm{d} \alpha\right)^{(1 / 4)} \\
& \times\left(\int_{\mathbb{R}}\left|S_{2}^{*}\left(\lambda_{2} \alpha\right)\right|^{2}\left|S_{k}\left(\lambda_{5} \alpha\right)\right|^{4} K_{\tau}(\alpha) \mathrm{d} \alpha\right)^{(1 / 4)} \\
& \ll\left(X^{(6 / 7)+2 \varepsilon}\right)^{(1 / 4)}\left(\tau X^{1+\varepsilon}\right)^{(3 / 8)}\left(\tau X^{(5 / 3)+\varepsilon}\right)^{(1 / 8)}\left(\tau X^{1+\varepsilon}\right)^{(1 / 4)}\left(\tau X^{(4 / k)+\varepsilon}\right)^{(1 / 4)} \\
& \ll \tau X^{(13 / 12)+(1 / k)-(1 / 28)+2 \varepsilon} \text {. }
\end{aligned}
$$

If $k=5$, then 


$$
\begin{aligned}
& \left|I\left(\tau, \eta, \mathfrak{m}_{1}\right)\right| \\
& \quad \ll\left(\sup _{\alpha \in \mathfrak{m}_{1}}\left|S_{1}\left(\lambda_{1} \alpha\right)\right|\right)^{(3 / 16)}\left(\int_{\mathbb{R}}\left|S_{1}\left(\lambda_{1} \alpha\right)\right|^{2} K_{\tau}(\alpha) \mathrm{d} \alpha\right)^{(13 / 32)} \\
& \quad \times\left(\int_{\mathbb{R}}\left|S_{3}\left(\lambda_{3} \alpha\right)\right|^{8} K_{\tau}(\alpha) \mathrm{d} \alpha\right)^{(3 / 32)}\left(\int_{\mathbb{R}}\left|S_{2}^{*}\left(\lambda_{2} \alpha\right)\right|^{2}\left|S_{4}\left(\lambda_{4} \alpha\right)\right|^{4} K_{\tau}(\alpha) \mathrm{d} \alpha\right)^{(1 / 4)} \\
& \quad \times\left(\int_{\mathbb{R}}\left|S_{2}^{*}\left(\lambda_{2} \alpha\right)\right|^{2}\left|S_{k}\left(\lambda_{5} \alpha\right)\right|^{6} K_{\tau}(\alpha) \mathrm{d} \alpha\right)^{(1 / 8)} \\
& \quad \times\left(\int_{\mathbb{R}}\left|S_{2}^{*}\left(\lambda_{2} \alpha\right) S_{3}\left(\lambda_{3} \alpha\right) S_{k}\left(\lambda_{5} \alpha\right)\right|^{2} K_{\tau}(\alpha) \mathrm{d} \alpha\right)^{(1 / 8)} \\
& \ll\left(X^{(6 / 7)+2 \varepsilon}\right)^{(3 / 16)}\left(\tau X^{1+\varepsilon}\right)^{(13 / 32)}\left(\tau X^{(5 / 3)+\varepsilon}\right)^{(3 / 32)}\left(\tau X^{1+\varepsilon}\right)^{(1 / 4)}\left(\tau X^{(6 / k)+\varepsilon}\right)^{(1 / 8)}\left(\tau X^{(2 / 3)+(2 / k)+\varepsilon}\right)^{(1 / 8)} \\
& \ll \\
& \ll X^{(13 / 12)+(1 / k)-(3 / 112)+2 \varepsilon} .
\end{aligned}
$$

In case $k=6$, we obtain

$$
\begin{aligned}
& \left|I\left(\tau, \eta, \mathfrak{m}_{1}\right)\right| \\
& \ll\left(\sup _{\alpha \in \mathfrak{m}_{1}}\left|S_{1}\left(\lambda_{1} \alpha\right)\right|\right)^{(1 / 6)}\left(\int_{\mathbb{R}}\left|S_{1}\left(\lambda_{1} \alpha\right)\right|^{2} K_{\tau}(\alpha) \mathrm{d} \alpha\right)^{(5 / 12)} \\
& \times\left(\int_{\mathbb{R}}\left|S_{3}\left(\lambda_{3} \alpha\right)\right|^{8} K_{\tau}(\alpha) \mathrm{d} \alpha\right)^{(1 / 12)}\left(\int_{\mathbb{R}}\left|S_{2}^{*}\left(\lambda_{2} \alpha\right)\right|^{2}\left|S_{4}\left(\lambda_{4} \alpha\right)\right|^{4} K_{\tau}(\alpha) \mathrm{d} \alpha\right)^{(1 / 4)} \\
& \times\left(\int_{\mathbb{R}}\left|S_{2}^{*}\left(\lambda_{2} \alpha\right)\right|^{2}\left|S_{k}\left(\lambda_{5} \alpha\right)\right|^{8} K_{\tau}(\alpha) \mathrm{d} \alpha\right)^{(1 / 12)} \\
& \times\left(\int_{\mathbb{R}}\left|S_{2}^{*}\left(\lambda_{2} \alpha\right) S_{3}\left(\lambda_{3} \alpha\right) S_{k}\left(\lambda_{5} \alpha\right)\right|^{2} K_{\tau}(\alpha) \mathrm{d} \alpha\right)^{(1 / 6)} \\
& \ll\left(X^{(6 / 7)+2 \varepsilon}\right)^{(1 / 6)}\left(\tau X^{1+\varepsilon}\right)^{(5 / 12)}\left(\tau X^{(5 / 3)+\varepsilon}\right)^{(1 / 12)}\left(\tau X^{1+\varepsilon}\right)^{(1 / 4)}\left(\tau X^{(8 / k)+\varepsilon}\right)^{(1 / 12)}\left(\tau X^{(2 / 3)+(2 / k)+\varepsilon}\right)^{(1 / 6)} \\
& \ll \tau X^{(13 / 12)+(1 / k)-(1 / 42)+2 \varepsilon} \text {. }
\end{aligned}
$$

It follows from (33)-(35) and (17) that

$$
\left|I\left(\tau, \eta, \mathfrak{m}_{1}\right)\right| \ll \tau^{2} X^{(13 / 12)+(1 / k)-\varepsilon} \ll \tau^{2} X^{(13 / 12)+(1 / k)} \mathscr{L}^{-2} .
$$

In order to establish an upper bound for $\left|I\left(\tau, \eta, \mathfrak{m}_{2}\right)\right|$ as small as possible, we need the following lemma.

Lemma 3 (Lemma 3.4 in [21]). Let

$$
\mathfrak{N}=\left\{\alpha: X^{(11 / 36)+2 \varepsilon}<\left|S_{3}\left(\lambda_{3} \alpha\right)\right| \leq X^{(1 / 3)}\right\} .
$$

§en, we have

$$
\int_{\Re}\left|S_{3}\left(\lambda_{3} \alpha\right)\right|^{2}\left|S_{4}\left(\lambda_{4} \alpha\right)\right|^{2} K_{\tau}(\alpha) \mathrm{d} \alpha \ll \tau X^{(1 / 6)+4 \varepsilon} .
$$

For $4 \leq k \leq 6$, by the Cauchy-Schwarz inequality, Lemmas 2 and 3, we obtain

$$
\begin{aligned}
& \left|I\left(\tau, \eta, \mathfrak{m}_{2}\right)\right| \ll X^{(1 / k)}\left(\sup _{\alpha \in \mathfrak{m}_{2}}\left|S_{2}^{*}\left(\lambda_{2} \alpha\right)\right|\right)\left(\int_{\mathfrak{N}}\left|S_{3}\left(\lambda_{3} \alpha\right)\right|^{2}\left|S_{4}\left(\lambda_{4} \alpha\right)\right|^{2} K_{\tau}(\alpha) d \alpha\right)^{(1 / 2)} \\
& \times\left(\int_{\mathbb{R}}\left|S_{1}\left(\lambda_{1} \alpha\right)\right|^{2} K_{\tau}(\alpha) d \alpha\right)^{(1 / 2)} \\
& \ll \tau X^{(1 / k)+(3 / 7)+(1 / 12)+(1 / 2)+4 \varepsilon} \\
& \ll \tau X^{(13 / 12)+(1 / k)-(1 / 14)+5 \varepsilon},
\end{aligned}
$$


where the trivial upper bound $S_{k}\left(\lambda_{5} \alpha\right) \ll X^{(1 / k)}$ is used. It is easily derived from (17) that

$$
\left|I\left(\tau, \eta, \mathfrak{m}_{2}\right)\right| \ll \tau^{2} X^{(13 / 12)+(1 / k)-\varepsilon} \ll \tau^{2} X^{(13 / 12)+(1 / k)} \mathscr{L}^{-2} .
$$

The upper bound estimation of $\left|I\left(\tau, \eta, \mathfrak{m}_{3}\right)\right|$ plays a crucial role in the proof. The parameter $\tau$, which is given by (17), is determined in this step. When $k=4$, by Hölder's inequality and Lemma 2, we have

$$
\begin{aligned}
\left|I\left(\tau, \eta, \mathfrak{m}_{3}\right)\right| & \ll\left(\sup _{\alpha \in \mathfrak{m}_{3}}\left|S_{3}\left(\lambda_{3} \alpha\right)\right|\right)\left(\int_{\mathbb{R}}\left|S_{1}\left(\lambda_{1} \alpha\right)\right|^{2} K_{\tau}(\alpha) d \alpha\right)^{(1 / 2)} \\
& \times\left(\left.\int_{\mathbb{R}}\left|S_{2}^{*}\left(\lambda_{2} \alpha\right)\right|\right|^{2}\left|S_{4}\left(\lambda_{4} \alpha\right)\right|^{4} K_{\tau}(\alpha) d \alpha\right)^{(1 / 4)} \\
& \times\left(\int_{\mathbb{R}}\left|S_{2}^{*}\left(\lambda_{2} \alpha\right)\right|^{2}\left|S_{k}\left(\lambda_{5} \alpha\right)\right|^{4} K_{\tau}(\alpha) d \alpha\right)^{(1 / 4)} \\
& \ll\left(X^{(11 / 36)+2 \varepsilon}\right)\left(\tau X^{1+\varepsilon}\right)^{(1 / 2)}\left(\tau X^{1+\varepsilon}\right)^{(1 / 4)}\left(\tau X^{(4 / k)+\varepsilon}\right)^{(1 / 4)} \\
& \ll \tau X^{(13 / 12)+(1 / k)-(1 / 36)+4 \varepsilon} .
\end{aligned}
$$

In the case of $k=5$, we obtain

$$
\begin{aligned}
& \left|I\left(\tau, \eta, \mathfrak{m}_{3}\right)\right| \ll\left(\sup _{\alpha \in \mathfrak{m}_{3}}\left|S_{2}^{*}\left(\lambda_{2} \alpha\right)\right|\right)^{(1 / 6)}\left(\sup _{\alpha \in \mathfrak{m}_{3}}\left|S_{3}\left(\lambda_{3} \alpha\right)\right|\right)^{(1 / 3)} \\
& \times\left(\int_{\mathbb{R}}\left|S_{1}\left(\lambda_{1} \alpha\right)\right|^{2} K_{\tau}(\alpha) \mathrm{d} \alpha\right)^{(1 / 2)}\left(\int_{\mathbb{R}}\left|S_{3}\left(\lambda_{3} \alpha\right)\right|^{8} K_{\tau}(\alpha) \mathrm{d} \alpha\right)^{(1 / 12)} \\
& \times\left(\int_{\mathbb{R}}\left|S_{2}^{*}\left(\lambda_{2} \alpha\right)\right|^{2}\left|S_{4}\left(\lambda_{4} \alpha\right)\right|^{4} K_{\tau}(\alpha) \mathrm{d} \alpha\right)^{(1 / 4)} \\
& \times\left(\int_{\mathbb{R}}\left|S_{2}^{*}\left(\lambda_{2} \alpha\right)\right|^{2}\left|S_{k}\left(\lambda_{5} \alpha\right)\right|^{6} K_{\tau}(\alpha) \mathrm{d} \alpha\right)^{(1 / 6)} \\
& \ll\left(X^{(3 / 7)+2 \varepsilon}\right)^{(1 / 6)}\left(X^{(11 / 36)+2 \varepsilon}\right)^{(1 / 3)}\left(\tau X^{1+\varepsilon}\right)^{(1 / 2)}\left(\tau X^{(5 / 3)+\varepsilon}\right)^{(1 / 12)} \\
& \times\left(\tau X^{1+\varepsilon}\right)^{(1 / 4)}\left(\tau X^{(6 / k)+\varepsilon}\right)^{(1 / 6)} \\
& \ll \tau X^{(13 / 12)+(1 / k)-(4 / 189)+4 \varepsilon} \text {. }
\end{aligned}
$$

If $k=6$, we deduce that

$$
\begin{aligned}
\left|I\left(\tau, \eta, \mathfrak{m}_{3}\right)\right| & \ll\left(\sup _{\alpha \in \mathfrak{m}_{3}}\left|S_{3}\left(\lambda_{3} \alpha\right)\right|\right)^{(2 / 3)}\left(\int_{\mathbb{R}}\left|S_{1}\left(\lambda_{1} \alpha\right)\right|^{2} K_{\tau}(\alpha) \mathrm{d} \alpha\right)^{(1 / 2)} \\
& \times\left(\int_{\mathbb{R}}\left|S_{2}^{*}\left(\lambda_{2} \alpha\right)\right|^{2}\left|S_{4}\left(\lambda_{4} \alpha\right)\right|^{4} K_{\tau}(\alpha) \mathrm{d} \alpha\right)^{(1 / 4)} \\
& \times\left(\int_{\mathbb{R}}\left|S_{2}^{*}\left(\lambda_{2} \alpha\right)\right|^{2}\left|S_{k}\left(\lambda_{5} \alpha\right)\right|^{8} K_{\tau}(\alpha) \mathrm{d} \alpha\right)^{(1 / 12)} \\
& \times\left(\int_{\mathbb{R}}\left|S_{2}^{*}\left(\lambda_{2} \alpha\right) S_{3}\left(\lambda_{3} \alpha\right) S_{k}\left(\lambda_{5} \alpha\right)\right|^{2} K_{\tau}(\alpha) \mathrm{d} \alpha\right)^{(1 / 6)} \\
& \ll\left(X^{(11 / 36)+2 \varepsilon}\right)^{(2 / 3)}\left(\tau X^{1+\varepsilon}\right)^{(1 / 2)}\left(\tau X^{1+\varepsilon}\right)^{(1 / 4)}\left(\tau X^{(8 / k)+\varepsilon}\right)^{(1 / 12)}\left(\tau X^{(2 / 3)+(2 / k)+\varepsilon}\right)^{(1 / 6)} \\
& \ll \tau X^{12}+\frac{1}{k}-\frac{1}{54}+4 \varepsilon
\end{aligned}
$$

Inequalities (41)-(43) and (17) together give

$$
\left|I\left(\tau, \eta, \mathfrak{m}_{3}\right)\right| \ll \tau^{2} X^{(13 / 12)+(1 / k)-\varepsilon} \ll \tau^{2} X^{(13 / 12)+(1 / k)} \mathscr{L}^{-2} .
$$

In the remainder of this section, we shall be trying to estimate $\left|I\left(\tau, \eta, \mathfrak{m}_{4}\right)\right|$. By a familiar dyadic dissection argument, we divide $\mathfrak{m}_{4}$ into at most $\ll \mathscr{L}^{3}$ disjoint sets $E\left(Z_{1}, Z_{2}, y\right)$. For $\alpha \in E\left(Z_{1}, Z_{2}, y\right)$, we have

$$
Z_{1}<\left|S_{1}\left(\lambda_{1} \alpha\right)\right| \leq 2 Z_{1}, Z_{2}<\left|S_{2}^{*}\left(\lambda_{2} \alpha\right)\right| \leq 2 Z_{2}, y<|\alpha| \leq 2 y
$$

where $\quad Z_{1}=2^{k_{1}} X^{(6 / 7)+2 \varepsilon}, Z_{2}=2^{k_{2}} X^{(3 / 7)+2 \varepsilon}, \quad$ and $y=2^{k_{3}} X^{-(1 / 8)}$ for some nonnegative integers $k_{1}, k_{2}$, and $k_{3}$. For the sake of convenience, we take the notation $\mathscr{A}$ as a shortcut for $E\left(Z_{1}, Z_{2}, y\right)$, and let $m(\mathscr{A})$ stand for the Lebesgue measure of $\mathscr{A}$.

Lemma 4 (Lemma 4.3 in [20]). We have

$$
m(\mathscr{A}) \ll y X^{(18 / 7)+9 \varepsilon} Z_{1}^{-2} Z_{2}^{-4} \text {. }
$$

When $k=4$, it follows from (11) and Hölder's inequality that 
Journal of Mathematics

7

$$
\begin{aligned}
\mid I & (\tau, \eta, \mathscr{A}) \mid \\
& \ll\left(\int_{\mathscr{A}}\left|S_{1}\left(\lambda_{1} \alpha\right)\right|^{2}\left|S_{2}^{*}\left(\lambda_{2} \alpha\right)\right|^{4} K_{\tau}(\alpha) \mathrm{d} \alpha\right)^{(1 / 12)}\left(\int_{\mathbb{R}}\left|S_{1}\left(\lambda_{1} \alpha\right)\right|^{2} K_{\tau}(\alpha) \mathrm{d} \alpha\right)^{(5 / 12)} \\
& \times\left(\int_{\mathbb{R}}\left|S_{3}\left(\lambda_{3} \alpha\right)\right|^{8} K_{\tau}(\alpha) \mathrm{d} \alpha\right)^{(1 / 8)}\left(\int_{\mathbb{R}}\left|S_{4}\left(\lambda_{4} \alpha\right)\right|^{16} K_{\tau}(\alpha) \mathrm{d} \alpha\right)^{(1 / 48)} \\
& \times\left(\int_{\mathbb{R}}\left|S_{k}\left(\lambda_{5} \alpha\right)\right|^{16} K_{\tau}(\alpha) \mathrm{d} \alpha\right)^{(1 / 48)}\left(\int_{\mathbb{R}}\left|S_{2}^{*}\left(\lambda_{2} \alpha\right)\right|^{2}\left|S_{4}\left(\lambda_{4} \alpha\right)\right|^{4} K_{\tau}(\alpha) \mathrm{d} \alpha\right)^{(1 / 6)} \\
& \times\left(\int_{\mathbb{R}}\left|S_{2}^{*}\left(\lambda_{2} \alpha\right)\right|^{2}\left|S_{k}\left(\lambda_{5} \alpha\right)\right|^{4} K_{\tau}(\alpha) \mathrm{d} \alpha\right)^{(1 / 6)} \\
\ll & \left(Z_{1}^{2} Z_{2}^{4} \cdot m(\mathscr{A}) \cdot \min \left(\tau^{2}, y^{-2}\right)\right)^{(1 / 12)}\left(\tau X^{1+\varepsilon}\right)^{(5 / 12)}\left(\tau X^{(5 / 3)+\varepsilon}\right)^{(1 / 8)} \\
& \times\left(\tau X^{3+\varepsilon}\right)^{(1 / 48)}\left(\tau X^{(12 / k)+\varepsilon}\right)^{(1 / 48)}\left(\tau X^{1+\varepsilon}\right)^{(1 / 6)}\left(\tau X^{(4 / k)+\varepsilon}\right)^{(1 / 6)} \\
\ll & \left(y X^{(18 / 7)+9 \varepsilon} \cdot \min \left(\tau^{2}, y^{-2}\right)\right)^{(1 / 12)} \tau^{(11 / 12)} X^{(41 / 48)+(11 / 12 k)+\varepsilon} \\
\ll & \tau X^{(13 / 12)+(1 / k)-(1 / 28)+2 \varepsilon},
\end{aligned}
$$

where Lemmas 2 and 3 are used.
When $k=5$, by the similar argument as in the proof of (47), we obtain

$$
\begin{aligned}
& |I(\tau, \eta, \mathscr{A})| \\
& \ll\left(\int_{\mathscr{A}}\left|S_{1}\left(\lambda_{1} \alpha\right)\right|^{2}\left|S_{2}^{*}\left(\lambda_{2} \alpha\right)\right|^{4} K_{\tau}(\alpha) \mathrm{d} \alpha\right)^{(1 / 16)}\left(\int_{\mathbb{R}}\left|S_{1}\left(\lambda_{1} \alpha\right)\right|^{2} K_{\tau}(\alpha) \mathrm{d} \alpha\right)^{(7 / 16)} \\
& \times\left(\int_{\mathbb{R}}\left|S_{3}\left(\lambda_{3} \alpha\right)\right|^{8} K_{\tau}(\alpha) \mathrm{d} \alpha\right)^{(1 / 8)}\left(\int_{\mathbb{R}}\left|S_{2}^{*}\left(\lambda_{2} \alpha\right)\right|^{2}\left|S_{4}\left(\lambda_{4} \alpha\right)\right|^{4} K_{\tau}(\alpha) \mathrm{d} \alpha\right)^{(1 / 8)} \\
& \times\left(\left.\int_{\mathbb{R}}|| S_{2}^{*}\left(\lambda_{2} \alpha\right)\right|^{2}\left|S_{4}\left(\lambda_{4} \alpha\right)\right|^{2}\left|S_{k}\left(\lambda_{5} \alpha\right)\right|^{4} K_{\tau}(\alpha) \mathrm{d} \alpha\right)^{(1 / 4)} \\
& \ll\left(Z_{1}^{2} Z_{2}^{4} \cdot m(\mathscr{A}) \cdot \min \left(\tau^{2}, y^{-2}\right)\right)^{(1 / 16)}\left(\tau X^{1+\varepsilon}\right)^{(7 / 16)} \\
& \times\left(\tau X^{(5 / 3)+\varepsilon}\right)^{(1 / 8)}\left(\tau X^{1+\varepsilon}\right)^{(1 / 8)}\left(\tau X^{(1 / 2)+(4 / k)+\varepsilon}\right)^{(1 / 4)} \\
& \ll\left(y X^{(18 / 7)+9 \varepsilon} \cdot \min \left(\tau^{2}, y^{-2}\right)\right)^{(1 / 16)} \tau^{(15 / 16)} X^{(43 / 48)+(1 / k)+\varepsilon} \\
& \ll \tau X^{(13 / 12)+(1 / k)-(3 / 112)+2 \varepsilon} \text {. }
\end{aligned}
$$

When $k=6$, we have

$$
\begin{aligned}
& |I(\tau, \eta, \mathscr{A})| \\
& \ll\left(\int_{\mathscr{A}}\left|S_{1}\left(\lambda_{1} \alpha\right)\right|^{2}\left|S_{2}^{*}\left(\lambda_{2} \alpha\right)\right|^{4} K_{\tau}(\alpha) \mathrm{d} \alpha\right)^{(1 / 16)}\left(\int_{\mathbb{R}}\left|S_{1}\left(\lambda_{1} \alpha\right)\right|^{2} K_{\tau}(\alpha) \mathrm{d} \alpha\right)^{(7 / 16)} \\
& \times\left(\int_{\mathbb{R}}\left|S_{3}\left(\lambda_{3} \alpha\right)\right|^{8} K_{\tau}(\alpha) \mathrm{d} \alpha\right)^{(1 / 8)}\left(\int_{\mathbb{R}}\left|S_{2}^{*}\left(\lambda_{2} \alpha\right)\right|^{2}\left|S_{4}\left(\lambda_{4} \alpha\right)\right|^{4} K_{\tau}(\alpha) \mathrm{d} \alpha\right)^{(1 / 4)} \\
& \times\left(\int_{\mathbb{R}}\left|S_{2}^{*}\left(\lambda_{2} \alpha\right)\right|^{2}\left|S_{k}\left(\lambda_{5} \alpha\right)\right|^{8} K_{\tau}(\alpha) \mathrm{d} \alpha\right)^{(1 / 8)} \\
& \ll\left(Z_{1}^{2} Z_{2}^{4} \cdot m(\mathscr{A}) \cdot \min \left(\tau^{2}, y^{-2}\right)\right)^{(1 / 16)}\left(\tau X^{1+\varepsilon}\right)^{(7 / 16)}\left(\tau X^{(5 / 3)+\varepsilon}\right)^{(1 / 8)}\left(\tau X^{1+\varepsilon}\right)^{(1 / 4)}\left(\tau X^{(8 / k)+\varepsilon}\right)^{(1 / 8)} \\
& \ll\left(y X^{(18 / 7)+9 \varepsilon} \cdot \min \left(\tau^{2}, y^{-2}\right)\right)^{(1 / 16)} \tau^{(15 / 16)} X^{(43 / 48)+(1 / k)+\varepsilon} \\
& \ll \tau X^{(13 / 12)+(1 / k)-(3 / 112)+2 \varepsilon} \text {. }
\end{aligned}
$$


Thanks to (17) and (47)-(49), we are led to the conclusion that

$$
\begin{aligned}
\left|I\left(\tau, \eta, \mathfrak{m}_{4}\right)\right| & \ll \mathscr{L}^{3} \cdot \max _{\mathscr{A}}|I(\tau, \eta, \mathscr{A})| \ll \tau^{2} X^{(13 / 12)+(1 / k)-\varepsilon} \\
& \ll \tau^{2} X^{(13 / 12)+(1 / k)} \mathscr{L}^{-2} .
\end{aligned}
$$

This together with (36), (40), (44), and (32) gives (30).

\section{The Trivial Regions}

Finally, it only remains to treat $I(\tau, \eta, \mathrm{t})$. Suppose that $r$ and $j$ are positive integers with $r \leq j$. For any $\xi \in\left[X^{\varepsilon},+\infty\right)$ and nonzero real $\lambda$, we have (see (5.1) and (5.2) in [20])

$$
\begin{aligned}
& \int_{\xi}^{+\infty}\left|S_{j}(\lambda \alpha)\right|^{2^{r}} K_{\tau}(\alpha) \mathrm{d} \alpha \ll \xi^{-1} X^{\left(\left(2^{r}-r\right) / j\right)+\varepsilon}, \\
& \int_{\xi}^{+\infty}\left|S_{2}^{*}\left(\lambda_{2} \alpha\right)\right|^{4} K_{\tau}(\alpha) \mathrm{d} \alpha \ll \xi^{-1} X^{1+\varepsilon} .
\end{aligned}
$$

It follows from Hölder's inequality that

$$
\begin{aligned}
|I(\tau, \eta, \mathrm{t})| \ll & \int_{\xi}^{+\infty}\left|S_{k}\left(\lambda_{5} \alpha\right) S_{2}^{*}\left(\lambda_{2} \alpha\right) \prod_{\substack{1 \leq j \leq 4 \\
j \neq 2}} S_{j}\left(\lambda_{j} \alpha\right)\right| K_{\tau}(\alpha) \mathrm{d} \alpha \\
\ll & \left(\int_{\xi}^{+\infty}\left|S_{1}\left(\lambda_{1} \alpha\right)\right|^{2} K_{\tau}(\alpha) \mathrm{d} \alpha\right)^{(1 / 2)}\left(\int_{\xi}^{+\infty}\left|S_{2}^{*}\left(\lambda_{2} \alpha\right)\right|^{4} K_{\tau}(\alpha) \mathrm{d} \alpha\right)^{(1 / 4)} \\
& \times\left(\int_{\xi}^{+\infty}\left|S_{3}\left(\lambda_{3} \alpha\right)\right|^{8} K_{\tau}(\alpha) \mathrm{d} \alpha\right)^{(1 / 8)}\left(\int_{\xi}^{+\infty}\left|S_{4}\left(\lambda_{4} \alpha\right)\right|^{16} K_{\tau}(\alpha) \mathrm{d} \alpha\right)^{(1 / 16)} \\
& \times\left(\int_{\xi}^{+\infty}\left|S_{k}\left(\lambda_{5} \alpha\right)\right|^{16} K_{\tau}(\alpha) \mathrm{d} \alpha\right)^{(1 / 16)} \\
\ll & \left(\xi^{-1} X^{1+\varepsilon}\right)^{(1 / 2)}\left(\xi^{-1} X^{1+\varepsilon}\right)^{(1 / 4)}\left(\xi^{-1} X^{(5 / 3)+\varepsilon}\right)^{(1 / 8)}\left(\xi^{-1} X^{3+\varepsilon}\right)^{(1 / 16)}\left(\xi^{-1} X^{(12 / k)+\varepsilon}\right)^{(1 / 16)} \\
\ll & \xi^{-1} X^{(55 / 48)+(3 / 4 k)+2 \varepsilon} .
\end{aligned}
$$

Recalling that $\xi=\tau^{-2} X^{(1 / 16)-(1 / 4 k)+10 \varepsilon}$ and inserting this expression into (52) yields

$$
|I(\tau, \eta, \mathfrak{t})| \ll \tau^{2} X^{(13 / 12)+(1 / k)-\varepsilon} \ll \tau^{2} X^{(13 / 12)+(1 / k)} \mathscr{L}^{-2} .
$$

\section{Completion of the Proof}

We are now in a position to get the desired conclusion. It should be noted that

$$
I(\tau, \eta, \mathbb{R})=I(\tau, \eta, \mathfrak{M})+I(\tau, \eta, \mathfrak{m})+I(\tau, \eta, \mathfrak{t}) .
$$

From this and (19), (30), and (53), we infer that $I(\tau, \eta, \mathbb{R}) \gg \tau^{2} X^{(13 / 12)+(1 / k)} \mathscr{L}^{-1}$. Hence, by $(15)$,

$$
\mathscr{N}(X) \gg \tau X^{(13 / 12)+(1 / k)} \mathscr{L}^{-5} .
$$

This implies inequality (16) has $\gg \tau X^{(13 / 12)+(1 / k)} \mathscr{L}^{-5}$ solutions in quintuples of primes $\left(p_{1}, p_{2}, \ldots, p_{5}\right)$ with $p_{2} \in \mathscr{I}, p_{5} \in I_{k}$, and $p_{j} \in I_{j}$, for $j \in\{1,3,4\}$. Notice that $\lambda_{1} / \lambda_{2}$ is irrational, $q$ is any denominator of a convergent to $\lambda_{1} / \lambda_{2}$ and $X=q^{7 / 3}$. By substituting (17) into (55), we deduce that $\mathcal{N}(X) \longrightarrow+\infty$ as $q \longrightarrow+\infty$. In view of

$$
\max _{1 \leq j \leq 5} p_{j}=X
$$

and (17), we obtain the required range of $\sigma(k)$ in Theorem 1 . This completes the proof of Theorem 1.

\section{Data Availability}

No data were used to support this study.

\section{Conflicts of Interest}

The authors declare that they have no conflicts of interest.

\section{Acknowledgments}

The work of the first author was supported by the Natural Science Basic Research Plan in Shaanxi Province of China (no. 2019JM-337), China Scholarship Council (CSC) Scholarship Program (no. 202008615008), and Scientific Research Foundation of Xi'an Polytechnic University (no. BS1508).

\section{References}

[1] K. F. Roth, "A problem in additive number theory," Proceedings of the London Mathematical Society, vol. 53, no. 2, pp. 381-395, 1951. 
[2] K. Thanigasalam, "On additive number theory," Acta Arithmetica, vol. 13, no. 3, pp. 237-258, 1968.

[3] K. Thanigasalam, "On sums of powers and a related problem," Acta Arithmetica, vol. 36, no. 2, pp. 125-141, 1980.

[4] K. Thanigasalam, "On certain additive representations of integers," Portugaliae Math, vol. 42, pp. 447-465, 1984.

[5] R. C. Vaughan, "On the representation of numbers as sums of powers of natural numbers," Proceedings of the London Mathematical Society, vol. 21, no. 3, pp. 160-180, 1970.

[6] R. C. Vaughan, "On sums of mixed powers," Journal of the London Mathematical Society, vol. 3, no. 2, pp. 677-688, 1971.

[7] J. Brüdern, "Sums of squares and higher powers ii," Journal of the London Mathematical Society, vol. 35, no. 2, pp. 244-250, 1987.

[8] J. Brüdern, "A problem in additive number theory," Mathematical Proceedings of the Cambridge Philosophical Society, vol. 103, no. 1, pp. 27-33, 1988.

[9] K. B. Ford, "The representation of numbers as sums of unlike powers," Journal of the London Mathematical Society, vol. 51, no. 2, pp. 14-26, 1995.

[10] K. B. Ford, "The representation of numbers as sums of unlike powers. II," Journal of the American Mathematical Society, vol. 9, no. 4, pp. 919-940, 1996.

[11] W. Schwarz, Survey of analogues of the Waring problem: diophantine inequalities, Additive Number Theory, Seminaire Theorie Des Nombres, Bordeaux, France, 1977.

[12] J. Brüdern, "Additive Diophantine inequalities with mixed powers II," Mathematika, vol. 34, no. 2, pp. 131-140, 1987.

[13] J. Brüdern, "The Davenport-Heilbronn Fourier transform method, and some diophantine inequalities," in Number Theory and Its Applications, S. Kanemitsu and K. Gÿory, Eds., pp. 59-87, Kluwer Academic Publishers, Dordrecht, The Netherlands, 1999.

[14] Z. Shan, "On a problem of the sums of powers of primes," Journal of University of Science and Technology of China, vol. 11, no. 4, pp. 1-13, 1981.

[15] K. Prachar, "Über ein problem vom Waring-Goldbach'schen typ II," Monatshefte Für Mathematik, vol. 57, no. 2, pp. 113-116, 1953, in German.

[16] X. M. Ren and K. M. Tsang, "Waring-Goldbach problem for unlike powers," Acta Mathematica Sinica, English Series, vol. 23, no. 2, pp. 265-280, 2007.

[17] W.X. Ge and W. P. Li, "One Diophantine inequality with unlike powers of prime variables," Journal of Inequalities and Applications, vol. 33, p. 8, 2016.

[18] Q. Mu, "One Diophantine inequality with unlike powers of prime variables," International Journal of Number Theory, vol. 13, no. 06, pp. 1531-1545, 2017.

[19] Z. Liu, "Diophantine approximation by unlike powers of primes," International Journal of Number Theory, vol. 13, no. 09, pp. 2445-2452, 2017.

[20] Q. Mu and Y. Qu, "A note on Diophantine approximation by unlike powers of primes," International Journal of Number Theory, vol. 14, no. 06, pp. 1651-1668, 2018.

[21] L. Zhu, "Diophantine inequality by unlike powers of primes," The Ramanujan Journal, vol. 51, no. 2, pp. 307-318, 2020.

[22] G. Gao and Z. Liu, "Results of Diophantine approximation by unlike powers of primes," Frontiers of Mathematics in China, vol. 13, no. 4, pp. 797-808, 2018.

[23] G. Harman, "The values of ternary quadratic forms at prime arguments," Mathematika, vol. 51, no. 1-2, pp. 83-96, 2004.

[24] G. Harman and A. V. Kumchev, "On sums of squares of primes," Mathematical Proceedings of the Cambridge Philosophical Society, vol. 140, no. 01, pp. 1-13, 2006.
[25] W. Ge, W. Li, and T. Wang, "On Diophantine approximation by unlike powers of primes," Open Mathematics, vol. 17, no. 1, pp. 544-555, 2019.

[26] H. Davenport and H. Heilbronn, "On indefinite quadratic forms in five variables," Journal of the London Mathematical Society, vol. 21, no. 3, pp. 185-193, 1946.

[27] R. C. Vaughan, The Hardy-Littlewood Method, Cambridge University Press, Cambridge, UK, 2nd edition, 1997.

[28] G. Harman, "Trigonometric sums over primes I," Mathematika, vol. 28, no. 2, pp. 249-254, 1981. 